\title{
Applying Axiomatic Design Theory to the Multi-objective Optimization of Disk Brake*
}

\author{
Zhiqiang Wu, Xianfu Cheng, and Junping Yuan \\ School of Mechanical and Electronical Engineering, East China \\ Jiaotong University, Nanchang 330013, China \\ chxf_xnesina.com
}

\begin{abstract}
The multi-objective optimization involves multiple, competing functionality requirements, which is mainly limited to downstream detailed design. Axiomatic design provides the theory to design a complex system top down and deals with multiple functional requirements (FR). It has demonstrated its strength in various types of design tasks. In fact, the objective function is a FR and those variables affecting the objective function are the design parameters (DPs). This paper presents an application of axiomatic design to multi-objective optimization. First, identify the relationship between FRs and DPs in terms of contribution of each DP to each FR by using orthogonal experiment and analysis of variance (ANOVA); then identify important design parameters to a FR and classify design variables into groups based on uncoupled design philosophy; and then establish the function dependence table, and sequentially optimize every objective function. An application in a disk brake design is used to demonstrate the use of the proposed method in dealing with real-world design problems. The results show that the proposed method provides a promising approach to optimize multiple, competing design objectives.
\end{abstract}

Keywords: Axiomatic design, Multi-objective optimization, ANOVA, Disk Brake.

\section{Introduction}

Multi-objective optimization is generally more difficult to achieve each objective optimum because of tradeoff between the various objectives, so the absolutely optimal solution may not exist. Axiomatic design approach has demonstrated its strength in various types of large-scale system design, including vehicles, aircrafts, manufacturing facilities, and so on [1]. Liu [2], Hwang [3] and Jeff [4] apply independent axiom of axiomatic design theory to improve and optimize multiobjective and large-scale engineering systems. In this paper, a systematic method is presented for applying independent axiom to multi-objective optimization problems. Firstly, identify the relationship between FRs and DPs in terms of contribution of

Foundation: Project supported by the National Natural Science Foundation of China (51165007). 
each DP to each FR by using orthogonal experiment and analysis of variance; secondly, important design parameters to a specific objective are identified and could be grouped into one set of parameters by means of the "uncoupled" philosophy of axiomatic design; then establish the function dependence table, and optimize every objective function in sequence.

\section{Multi-objective Optimization Formulation}

The mathematical model of multi-objective optimization is generally expressed as

$$
\begin{gathered}
\text { Min: } F(\mathbf{x})=\left\{f_{1}(\mathbf{x}), f_{2}(\mathbf{x}) ; \cdots, f_{m}(\mathbf{x})\right\}^{\mathrm{T}} \\
\text { s.t. } \quad g_{j}(\mathbf{x}) \leq 0 \quad j=1, \cdots, q \\
h_{k}(\mathbf{x})=0 \quad k=1, \cdots, p
\end{gathered}
$$

where, $\mathbf{x}=\left(x_{1}, x_{2}, \ldots, x_{n}\right)^{\mathrm{T}}$ is $n$-dimensional design variables; $\mathrm{F}(\mathrm{x})$ is the vector of objective functions; $f_{i}(\mathbf{x})$ is the $i$ th sub-objective function and $\mathrm{m}$ is the number of subobjective function; $g_{i}(\mathbf{x})$ is the $j$ th inequality constraint and $q$ is the number of inequality constraint functions, $h_{k}(\mathbf{x})$ is the $k$ th equality constraints and $k$ is the number of equality constraint functions.

In the above equation, achieving the optimum of each objective is generally difficult. Specially, when there are tradeoffs between the various objectives, namely, the optimization problem has conflicting goals, it is impossible to make each subobjective simultaneously attain optimum. Thus, it should be required that the optimal solutions of all sub-objectives are balanced, so that the totally satisfactory solutions can be obtained. Compared to single objective optimization, the theory and computational methods on multi-objective optimization are not perfect. Therefore, it is necessary to introduce other relative design theories, such as axiomatic design, to further promote multi-objective optimization theory and methodology.

\section{Optimization Design Based on "Uncoupled" Philosophy}

In fact, the objective function is a FR and those variables affecting the objective function are the DPs already mentioned. But, in this case, the FR must be able to be expressed by the DPs in the form of a mathematical equation. It is obvious that optimization design is the method mapping between one FR and a set of DPs at a lower or the lowest level of FR and DP hierarchies in the process of axiomatic design [5]. For the design with only a FR, FR is clearly independent, and the optimal value of FR can be easily obtained by adjusting the corresponding DPs. Here, optimal design is very effective. For those with multiple objective functions, it is difficult to tune the corresponding DPs to simultaneously obtain the optimal solutions of FRs. For example, in order to obtain the optimum of FR1, the value of DPs should be tuned. In order to improve the FR2, the value of DPs should be further tuned, but the 
FR1 will be changed. Therefore, it is needed to return to tune the DPs to optimize FR1; on the contrary, the FR2 will be changed. So again, this is a trade-off among multiple objectives.

In the axiomatic design, uncoupled or decoupled design is a good design [1]. The functional forms of the relations with two functional requirements are as follows:

For uncoupled design: $f_{1}=f_{1}\left(x_{1}\right), f_{2}=f_{2}\left(x_{2}\right)$

For decoupled design: $f_{1}=f_{1}\left(x_{1}\right), f_{2}=f_{2}\left(x_{1}, x_{2}\right)$

where $f_{1}$ and $f_{2}$ are functional requirements and $x_{1}$ and $x_{2}$ are corresponding design parameters. For uncoupled design, the design parameters can be determined separately. For decoupled design, $x_{1}$ and $x_{2}$ should be determined sequentially. In the first step of the decoupled design, the value of $x_{1}$ can be get by optimizing $f_{1}$, and $x_{1}$ is fixed in the next step as $x_{1}{ }^{*}$. In the second step, the value of $x_{2}$ can be obtained by optimizing $f_{2}$. The solution obtained from the above process could not be the optimum when $x_{1}$ is not fixed in the second step. Therefore, the decoupled design may not be good one when the design solution is calculated by a mathematical optimization. It is necessary to make the design to be uncoupled design.

In the multi-objective optimization problems, there are usually two different solving methods. The one is that the most important FR is selected as an objective function and the other FRs are ignored. Thus multiple FRs are transformed into single $\mathrm{FR}$, and optimization is effective to determine the optimal value of FR and the value of corresponding DPs, but the other FRs can't be optimized. The other is that every FR is weighted to form the aggregate objective function. Although it is also single FR optimization, it can't optimize FRs directly. Additionally, it is difficult to identify the weight. Therefore, in order to satisfy the independence axiom, FRs should be redefined or DPs should be reselected, and the different FRs is controlled by corresponding DPs independently. For uncoupled design, each sub-objective of multiobjective optimization is independent mutually, which is equivalent to single objective optimization.

For coupled design problem: $f_{1}=f_{1}\left(x_{1}, x_{2}\right), f_{2}=f_{2}\left(x_{1}, x_{2}\right)$. If the influence of $x_{1}$ on $f_{1}$ is far greater than that of $x_{2}$ on $f_{1}$, and the influence of $x_{2}$ on $f_{2}$ is far greater than that of $x_{1}$ on $f_{2}$, the problem can be considered as a nearly uncoupled problem. Thus the axiomatic design process can be applied. When the influence of design parameters on the rest of design objectives can't be ignored, it is a strong coupling problem that can't be dealt with by axiomatic design.

Generally, there are more design variables than objective functions in optimization design. However, according to axiomatic design theory, the number of design variables (design parameters) must be the same as that of the objective functions (functional requirements in axiomatic design). Therefore, these designs are generally redundant, or may be coupled, and they violate the independence axiom and are not good designs. When the number of design variables is large, the variables can be grouped to have similar characteristics via the sensitivity information. That is, important variables to a specific objective function can be grouped into one set of parameters, and the number of the groups is the same as that of the objective functions. Thus the design is rearranged to be a nearly uncoupled design. The pertinence is backed up by the Theorem 8 in axiomatic design theory. 
Theorem 8: Independence and Tolerance.

A design is an uncoupled design when the designer-specified tolerance is greater than

$$
\Delta R_{i}>\left(\sum_{\substack{j=1 \\ j \neq i}}^{n}\left(\partial R_{i} / \partial P_{j}\right) \Delta P_{j}\right) i=1,2, \cdots, n .
$$

in which case the non-diagonal elements of the design matrix can be neglected from design consideration.

A multi-objective optimization problem will be transformed into single objective optimization problem in sequence by utilizing the method of large-scale optimization system or decomposition and coordination method of multi-disciplinary optimization. As the optimal solution of each sub-objective function may be not the optimum of multi-objective optimization, among of sub-objective function should be coordinated. According to optimization method on decomposition and coordination, the design variable of the previous objective function is fixed in the next objective function in the optimization process; the next objective function only optimize those design variables that is classified into this group, and the optimal variables will be returned to the previous objective function. By an iterative manner again and again, the optimization process continues until it satisfies the convergence condition. The key question of the "decomposition/coordination" optimization is how to identify the corresponding design variables and constraints of each objective function. In largescale / multi-disciplinary optimization, the design variables are grouped according to the locations of parts in structural design or experts-based domain knowledge. In this research, the decomposition is based on the relationship between DPs and FRs, which is defined as "logical decomposition".

\section{Optimization Design Method Using Independent Axiom}

The steps of multi-objective optimization design using independent axiom in axiomatic design can be given as in the following:

Step 1. Select design variables and identify objective functions and constraints.

Consider an optimization design problem that consists of six design variables, three objective functions and three constraints functions which is given by the following design equation.

$$
\begin{array}{ll}
\text { Min } & F(\mathbf{X})=\operatorname{Min}\left\{f_{1}(\mathbf{x}), f_{2}(\mathbf{x}), f_{3}(\mathbf{x})\right\}^{\mathrm{T}} \\
\text { s.t. } & g_{1}\left(x_{1}, x_{2}\right) \leq 0 \\
& g_{2}\left(x_{1}, x_{4}, x_{5}\right) \leq 0 \\
& g_{3}\left(x_{1}, x_{3}, x_{5}, x_{6}\right) \leq 0
\end{array}
$$

The vector of design variables is $\mathbf{x}=\left\{x_{1}, x_{2}, x_{3}, x_{4}, x_{5}, x_{6}\right\}^{\mathrm{T}}$. 
Step 2. Put the optimization objectives and design variables into a design matrix. The relationship between design variables and optimization objectives is expressed as

$$
\left[\begin{array}{l}
\text { FR } 1 \\
\text { FR2 } \\
\text { FR3 }
\end{array}\right]=\left[\begin{array}{llllll}
1 & 1 & 1 & 1 & 0 & 0 \\
1 & 0 & 0 & 1 & 1 & 1 \\
0 & 1 & 1 & 1 & 1 & 1
\end{array}\right] \cdot\left[\begin{array}{c}
x_{1} \\
x_{2} \\
x_{3} \\
x_{4} \\
x_{5} \\
x_{6}
\end{array}\right]
$$

where FRi is the $i$ th functional requirement, namely design objective. The sign "1" means a relation exists and " 0 " means there is no relation between FR and design variable.

If the design matrix is rearranged into a new matrix by making it as diagonal matrix as possible, it shows the optimization objectives are independent, then turn to step 4 .

Step 3. Use orthogonal experiment and ANOVA to identify the contribution of each design variables to objective functions, and determine the principal design variables and ignore those design variables with small influence. In design matrix, the sign " $X$ " means design variable has significant effect on FR and "+" means design variable has little effect on FR. The equation (4) is rewritten as

$$
\left[\begin{array}{l}
\text { FR1 } \\
\text { FR2 } \\
\text { FR3 }
\end{array}\right]=\left[\begin{array}{cccccc}
\times & \times & + & + & 0 & 0 \\
+ & 0 & 0 & \times & \times & + \\
0 & + & \times & + & + & \times
\end{array}\right] \cdot\left[\begin{array}{c}
x_{1} \\
x_{2} \\
x_{3} \\
x_{4} \\
x_{5} \\
x_{6}
\end{array}\right]
$$

Rearrange the design matrix to make it as diagonal matrix as possible.

$$
\left[\begin{array}{l}
\text { FR1 } \\
\text { FR2 } \\
\text { FR3 }
\end{array}\right]=\left[\begin{array}{cccccc}
\times & \times & 0 & + & + & 0 \\
+ & 0 & \times & \times & 0 & + \\
0 & + & + & + & \times & \times
\end{array}\right] \cdot\left[\begin{array}{c}
x_{1} \\
x_{2} \\
x_{4} \\
x_{5} \\
x_{3} \\
x_{6}
\end{array}\right]
$$

The design variables are grouped and the number of the groups is the same as that of optimization objectives. Thus, the design will be nearly uncoupled design. According 
to equation (6), the design variables are classified into three groups. The design parameters DP1 include $\mathrm{x} 1$ and $\mathrm{x} 2$, DP2 include $\mathrm{x} 4$ and $\mathrm{x} 5$, and DP3 include $\mathrm{x} 3$ and $\mathrm{x} 6$. Then the formula can be rewritten as

$$
\left[\begin{array}{l}
\text { FR1 } \\
\text { FR2 } \\
\text { FR3 }
\end{array}\right]=\left[\begin{array}{lll}
\times & + & + \\
+ & \times & + \\
+ & + & \times
\end{array}\right]\left[\begin{array}{c}
\mathrm{DP} 1\left(x_{1}, x_{2}\right) \\
\mathrm{DP} 2\left(x_{4}, x_{5}\right) \\
\mathrm{DP} 3\left(x_{3}, x_{6}\right)
\end{array}\right]
$$

Step 4. Suppose $x_{p}$ and $x_{q}$ lie in the same group $f_{i}(\mathbf{x})$, if one constraint is only relative to $x_{p}$ and $x_{q}$, this constraint will be classified into this group. All other constraints are classified into different groups by analogy, and then go to step 6. Otherwise, go to the next step.

Step 5. According to the relationship between the design functions (including the objective function and constraint functions) and design variables, establish the dependence matrix on the design functions and design variables, which is named as the function dependence table (FDT). If a constraint function relates to one design variable, the corresponding position in FDT is marked as " 1 ", otherwise marked as " 0 ". Here, the contribution values of design variables on the constraint function are not considered. The relationship between the objective function and design variables is still marked as " $x$ " or "+".

Table 1. Function Dependence Table

\begin{tabular}{lcccccc}
\hline & $x_{1}$ & $x_{2}$ & $x_{4}$ & $x_{5}$ & $x_{3}$ & $x_{6}$ \\
\hline$f_{1}$ & $\times$ & $\times$ & + & + & & \\
$g_{1}$ & 1 & 1 & & & & \\
$g_{2}$ & 1 & & & 1 & & \\
$g_{3}$ & 1 & & & 1 & 1 & 1 \\
$f_{2}$ & + & & $\times$ & $\times$ & & + \\
$g_{2}$ & 1 & & 1 & 1 & & \\
$g_{3}$ & 1 & & & 1 & 1 & 1 \\
$f_{3}$ & & + & + & + & $\times$ & $\times$ \\
$g_{3}$ & 1 & & & 1 & 1 & 1 \\
\hline
\end{tabular}

In any case, the constraints must be met, so they can be classified into the different groups. Namely, if a constraint relates to design variables of multiple groups, it can be respectively classified into those groups. As shown in table $1, g_{1}(\mathbf{x})$ only relate to $\left\{f_{1}(\mathbf{x}) ; x_{1}, x_{2}\right\}$, it will be classified into this group; $g_{3}(\mathbf{x})$ relate to design variables of three groups $\left\{x_{1} ; x_{5} ; x_{3}, x_{6}\right\}$, it will be respectively classified into these groups.

Step 6. If the design functions of each group are independent, the multi-objective optimization problem can be solved by single objective optimization manner. Then go to step 10. However, in many cases, even if the objective functions are independent, the constraint functions are often closely linked to each other. So, it can not be solved in single objective optimization manner and should be optimized in sequence. 
Step 7. Determine the order of each group and optimize them in sequence. According to the order of optimization, if the optimization objective and constraints in one group not only relate to the design variables in this group, but also they relate to that in other group, the design variables in this group is only optimized and that in other group will be fixed. In the next group, the values of design variables obtained from the previous group are fixed. Each group is optimized in sequence, and set $\mathrm{k}=1$.

Step 8. Start from the first group continuously, the design variables in other groups are fixed. The other groups will be optimized in sequence and set $\mathrm{k}=\mathrm{k}+1$.

Step 9. Determine the optimization results whether meet the convergence criteria. If $\left|\left(\mathbf{x}^{k+1}-\mathbf{x}^{k}\right) / \mathbf{x}^{k+1}\right|<\varepsilon$, then return to step 10 , where $\varepsilon$ is the convergence precision and is usually set $\varepsilon=10^{-3} \sim 10^{-4}$. Otherwise return to step 8 and continue for the next cycle.

Step 10. Output the optimal solution $\left(\mathbf{x}^{*}, F^{*}\right)$.

The algorithm flow of optimization design using "uncoupled" idea of axiomatic design is shown in figure 1.

\section{Case Study}

In the optimization design for caliper disc brake used in automobile, the short braking time and small nave parameter under controllable temperature are required. When the vehicle is full-loaded the load of a single wheel $W$ is $3400 \mathrm{~N}$; the vehicle's running speed $v$ is $160 \mathrm{~km} / \mathrm{h}$; the radius of a wheel $r$ is $350 \mathrm{~mm}$; the number of brakes (i.e. number of wheels) $m$ is 4 ; the allowed maximum diameter for brake disk $\left[D_{\max }\right]$ is $300 \mathrm{~mm}$; the allowed maximum temperature $\left[T_{\max }\right]$ is $260^{\circ} \mathrm{C}$; the material of brake disc is steel and the original temperature $T_{i}$ is $35^{\circ} \mathrm{C}$; the diameter of nave $D_{h}$ is $75 \mathrm{~mm}$; the friction coefficient between pad and brake disc $\mu$ is 1 and the adhesion coefficient between tire and road surface $\varphi$ is 1 ; the allowed maximum pressure of pad $\left[p_{\max }\right]$ is $3 \mathrm{MPa}$; the allowed maximum oil pressure of hydro-cylinder $\left[p_{0 \max }\right]$ is $7 \mathrm{MPa}$; the thickness of hydro-cylinder wall $t_{c}$ is $6.5 \mathrm{~mm}$.

The structural relationship between caliper and brake disc is shown in fig. 2. The circular friction surface of pad is discretized as an arc concentric with the disc, as shown in fig. 3. Considering that asymmetrical wear process lead to $p v^{\prime}$ (unit-pressure $\times$ Rotate-speed) become balance gradually on the whole fiction plane, so we suppose that $p v^{\prime}=C=$ const .

The force of pad acting on disc:

$$
F=\int_{R-d / 2}^{R+d / 2} \frac{C}{r} l d r
$$

where $l=r \cdot \beta=2 r \cdot \cos ^{-1}\left(\frac{R^{2}+r^{2}-(d / 2)^{2}}{2 R r}\right)$. 


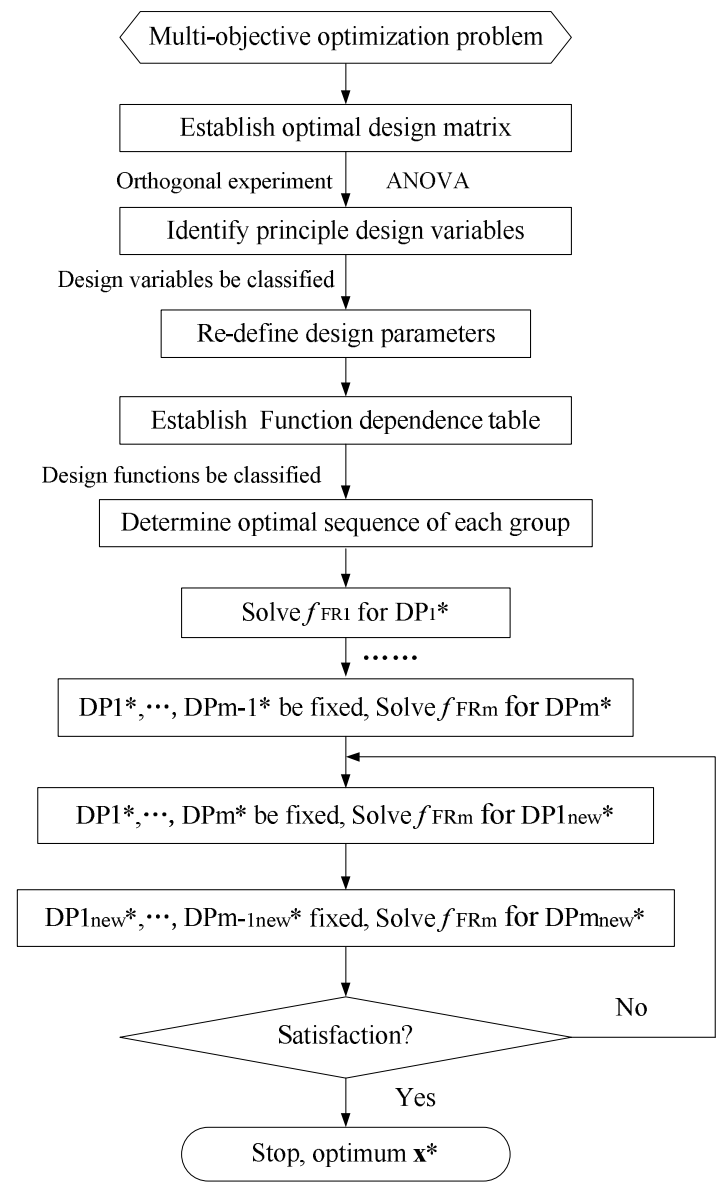

Fig. 1. The flow diagram of optimization design using "uncoupled" idea of axiomatic design

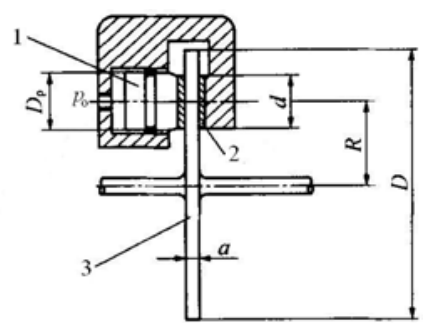

1. piston 2. pad 3. disc

Fig. 2. The structure diagram of disk brake

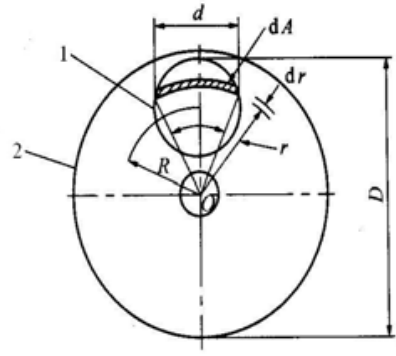

1. pad 2. pad disc

Fig. 3. Calculation diagram of brake 
The friction torque of braking:

$$
T_{f}=\int_{R-d / 2}^{R+d / 2} \mu p r d A=2 \mu F I_{2}
$$

where $I_{2}=\int_{R-d / 2}^{R+d / 2} \frac{l}{I_{1}} d r, \quad I_{1}=\int_{R-d / 2}^{R+d / 2} \frac{l}{r} d r$.

During the process of breaking the power dissipation of pad and brake disc is as much as kinetic energy of auto vehicle. Then the braking time can be derived:

$$
t=\frac{W_{a} \cdot v^{2}}{4 \pi F \mu I_{2} \cdot n_{0} \cdot m g} .
$$

where $W_{a}$ is total weight of a car $(\mathrm{N}) ; n_{0}$ is the rotation speed of brake discs or wheels when start braking (r/min); $F$ is thrust force of oil cylinder, $F=\frac{\pi}{4} D_{p}{ }^{2} \cdot p_{0}$, where $D_{\mathrm{p}}$ is diameter of piston $(\mathrm{mm}) ; p_{0}$ is oil pressure (MPa)

\subsection{Mathematical Model}

(1) Design variables

$$
\mathbf{x}=\left\{x_{1}, x_{2}, x_{3}, x_{4}, x_{5}, x_{6}\right\}^{\mathrm{T}}=\left\{R, d, D, D_{p}, a, p_{0}\right\}^{\mathrm{T}} \text {. }
$$

The design variables are shown in fig. 2 and fig. 3 .

(2) Constraint functions

$$
\left\{\begin{array}{l}
g_{1}(\mathbf{x})=-x_{1}+1 / 2 x_{2}+1 / 2 D_{\mathrm{h}} \leq 0 \\
g_{2}(\mathbf{x})=x_{1}+1 / 2 x_{2}-1 / 2 x_{3} \leq 0 \\
g_{3}(\mathbf{x})=-x_{1}+1 / 2 x_{4}+t_{c}+1 / 2 D_{\mathrm{h}} \leq 0 \\
g_{4}(\mathbf{x})=x_{3}-\left[D_{\max }\right] \leq 0 \\
g_{5}(\mathbf{x})=x_{6}-\left[p_{0 \max }\right] \leq 0 \\
g_{6}(\mathbf{x})=\frac{\pi x_{4}^{2} \cdot x_{6}}{4 I_{1}\left(x_{1}-x_{2} / 2\right)}-\left[p_{\max }\right] \leq 0 \\
g_{7}(\mathbf{x})=\mu \pi D_{p}^{2} p_{0} I_{2} / 2-W r \varphi \leq 0 \\
g_{8}(\mathbf{x})=\frac{4 E}{J \pi c \rho x_{3}^{2} x_{5}}+T_{i}-T_{\max } \leq 0
\end{array}\right.
$$

where $J$ is mechanical equivalent of heat and equals to $1 \mathrm{~N} \cdot \mathrm{m} / \mathrm{J}$; c is specific heat capacity and equals to $472.8 \mathrm{~J} / \mathrm{kg}^{\circ} \mathrm{C}$; $\rho$ is density and equals to $7.8 \times 10-6 \mathrm{~kg} / \mathrm{mm}^{3} ; E$ is power dissipation of friction torque and equals to $W_{a} \cdot v^{2} /(2 m g)$.

(3) Objective functions

To assure the safety of vehicle, it is essential to improve the work efficiency of brakes and to shorten the braking time, so minimizing braking time should be 
considered as optimization objective. In addition, minimizing thickness and temperature of the brake disc can be also considered as other two objectives. i.e.:

$$
\operatorname{Min} F(\mathbf{x})=\operatorname{Min}\left\{f_{1}(\mathbf{x}), f_{2}(\mathbf{x}), f_{3}(\mathbf{x})\right\}^{\mathrm{T}} .
$$

where $f_{1}(\mathbf{x})=t, f_{2}(\mathbf{x})=a, f_{3}(\mathbf{x})=\frac{4 E}{J \pi c \rho x_{3}^{2} x_{5}}$.

\subsection{Computing Process and Results}

(1) Identify the relationship between optimization objectives and design variables in terms of design matrix, and then rearrange the design matrix to make it into a nearly diagonal matrix or triangular matrix.

$$
\left[\begin{array}{l}
\mathrm{FR} 1 \\
\mathrm{FR} 2 \\
\mathrm{FR} 3
\end{array}\right]=\left[\begin{array}{llllll}
1 & 1 & 1 & 1 & 0 & 0 \\
0 & 0 & 0 & 0 & 1 & 0 \\
0 & 0 & 0 & 0 & 1 & 1
\end{array}\right] \cdot\left[\begin{array}{c}
x_{1} \\
x_{2} \\
x_{4} \\
x_{6} \\
x_{5} \\
x_{3}
\end{array}\right]
$$

As FR1 is independent of FR2 and FR3, and FR2 is only related to $x_{5}$, so we only need to determine the contribution of $x_{3}$ and $x_{5}$ to FR3. Since there are three subobjectives in optimization design, the design variables can be grouped and the number of the groups is three.

Table 2. Level of factor (unit: $\mathrm{mm}$ )

\begin{tabular}{cccc}
\hline \multirow{3}{*}{ Factor } & \multicolumn{3}{c}{ Level } \\
\cline { 2 - 4 } & 1 & 2 & 3 \\
\hline$x_{3}$ & 200 & 250 & 300 \\
$x_{5}$ & 4 & 5 & 6 \\
\hline
\end{tabular}

(2) Identify the contribution of $x_{3}$ and $x_{5}$ to FR3 using orthogonal experiment and ANOVA. Three levels are determined for both $x_{3}$ and $x_{5}$, as shown in table 2, and the orthogonal table $L_{9}\left(3^{4}\right)$ is selected.

The results of ANOVA show that $x_{3}$ has great influence on FR3 and $x_{5}$ has small influence on FR3. Then the equation (13) can be rewritten as

$$
\left[\begin{array}{l}
\text { FR1 } \\
\text { FR2 } \\
\text { FR3 }
\end{array}\right]=\left[\begin{array}{lll}
1 & 0 & 0 \\
0 & 1 & 0 \\
0 & + & \times
\end{array}\right]\left[\begin{array}{c}
\mathrm{DP} 1\left(x_{1}, x_{2}, x_{4}, x_{6}\right) \\
\mathrm{DP} 2\left(x_{5}\right) \\
\mathrm{DP} 3\left(x_{3}\right)
\end{array}\right] .
$$


(3) Establish the function dependence table. The FDT of disc brake is shown in Table 3.

Table 3. FDT of disc brake

\begin{tabular}{ccccccc}
\hline & $x_{1}$ & $x_{2}$ & $x_{4}$ & $x_{6}$ & $x_{5}$ & $x_{3}$ \\
\hline$f_{1}$ & 1 & 1 & 1 & 1 & & \\
$g_{1}$ & 1 & 1 & & & & \\
$g_{2}$ & 1 & 1 & & & & 1 \\
$g_{3}$ & 1 & & 1 & & & \\
$g_{5}$ & & & & 1 & & \\
$g_{6}$ & 1 & 1 & 1 & 1 & & \\
$g_{7}$ & 1 & 1 & 1 & 1 & & \\
$f_{2}$ & + & & & & 1 & \\
$g_{8}$ & & & & & 1 & 1 \\
$f_{3}$ & & & & & + & $\times$ \\
$g_{2}$ & 1 & 1 & & & & 1 \\
$g_{4}$ & & & & & & 1 \\
$g_{8}$ & & & & & 1 & 1 \\
\hline
\end{tabular}

(4) According to table $3, f_{1}(\mathbf{x})$ is independent of $f_{2}(\mathbf{x})$ and the relation between $f_{3}(\mathbf{x})$ and $f_{2}(\mathbf{x})$ is nearly uncoupled. Thus, $f_{1}(\mathbf{x})$ and $f_{2}(\mathbf{x})$ can be optimized simultaneously. But both the constraint $g_{2}$ in the group $\left\{f_{1}(\mathbf{x})\right\}$ and $g_{8}$ in the group $\left\{f_{2}(\mathbf{x})\right\}$ relate to $x_{3}$ in the group $\left\{f_{3}(\mathbf{x})\right\}, x_{3}$ should be fixed in the optimization process of $f_{1}(\mathbf{x})$ and $f_{2}(\mathbf{x})$. When $f_{3}(\mathbf{x})$ is optimized, $x_{1}, x_{2}$ and $x_{5}$ should be fixed.

Continue the next iteration until convergence criterion is satisfied. Then the optimal solutions are obtained.

The initial sizes of disc brake and the results of the weighted optimization method and the proposed method in this paper are summarized in table 4, here weighting factors $w_{1}, w_{2}$ and $w_{3}$ are $0.495,0.495$ and 0.01 respectively. According to the results and process of calculation, it shows that results obtained from the weighted optimization method will change with different weighting factors, so it is difficult to balance every objective. The optimal results based on the proposed method are more satisfactory, which consider the influence of design variables to design objectives and make the design to be nearly uncoupled design. The values of the first two objectives are smaller except the third objective, which is consistent with the importance of the first two objectives.

Table 4. The optimal results

\begin{tabular}{|c|c|c|c|c|c|c|c|c|c|}
\hline & $x_{1}(\mathrm{~mm})$ & $x_{2}(\mathrm{~mm})$ & $x_{3}(\mathrm{~mm})$ & $x_{4}(\mathrm{~mm})$ & $x_{5}(\mathrm{~mm})$ & $x_{6}(\mathrm{MPa})$ & $\begin{array}{c}f_{1}(\mathbf{x})=t \\
(\mathrm{~s})\end{array}$ & $\begin{array}{c}f_{2}(\mathbf{x})=a \\
(\mathrm{~mm})\end{array}$ & $\begin{array}{c}f_{3}(\mathbf{x})=T \\
\left({ }^{\circ} \mathrm{C}\right)\end{array}$ \\
\hline Initial value & 100 & 60 & 280 & 40 & 5 & 3 & 7.241 & 5 & 378.938 \\
\hline $\begin{array}{c}\text { Weighted } \\
\text { optimization }\end{array}$ & 100.016 & 60.036 & 280.145 & 40.077 & 7.272 & 3.516 & 6.153 & 7.272 & 207.484 \\
\hline $\begin{array}{l}\text { Proposed } \\
\text { method }\end{array}$ & 100.052 & 60.121 & 300 & 40.258 & 5.848 & 4.727 & 4.535 & 5.848 & 224.997 \\
\hline
\end{tabular}




\section{Conclusions}

In this paper, a new method of multi-objective optimization is presented. The concept of contribution of design variables to design objectives is used to identify the relation between them. Following the "uncoupled design" idea of axiomatic design, the design matrix is rearranged to be as diagonal matrix as possible. If the design is a nearly uncoupled design, an iterative method is suggested. The important design variables to a specific objective are identified and can be grouped into one set of parameters, and then establish the function dependence table and optimize every objective function in sequence. The optimization design of disc brake has been solved to show the validity of the proposed method.

\section{References}

1. Suh, N.P.: Axiomatic design: advances and applications. Oxford University Press, New York (2001)

2. Liu, X.P., Soderborg, N.: Improving an existing design based on axiomatic design principles. In: First International Conference on Axiomatic Design, Cambridge, U.S., June 21-23, pp. 199-202 (2000)

3. Hwang, K.H., Lee, K.W., Park, G.J., et al.: Robust design of the vibratory gyroscope with unbalanced inner torsion gimbal using axiomatic design. In: Second International Conference on Axiomatic Design, pp. 1-7. Massachusetts Institution of Technology, Boston (2002)

4. Jeff, T., Ge, P.: Applying axiomatic design theory to the evaluation and optimization of large-scale engineering systems. Journal of Engineering Design 17(1), 1-16 (2006)

5. Chen, K.Z.: Identifying the relationship among design methods-key to successful applications and developments of design methods. Journal of Engineering Design 10, 125-141 (1999) 